\title{
THREE NEW COOL BROWN DWARFS DISCOVERED WITH THE WIDE-FIELD INFRARED SURVEY EXPLORER (WISE) AND AN IMPROVED SPECTRUM OF THE Y0 DWARF WISE J041022.71+150248.4
}

\author{
Michael C. Cushing ${ }^{1}$, J. Davy Kirkpatrick ${ }^{2}$, Christopher R. Gelino ${ }^{2}$, Gregory N. MaCe ${ }^{3}$, \\ Michael F. SKRUTSKIE ${ }^{4}$, AND ANDREW GOULD ${ }^{5}$ \\ ${ }^{1}$ Department of Physics and Astronomy, The University of Toledo, 2801 West Bancroft Street, Toledo, OH 43606, USA; michael.cushing@utoledo.edu \\ ${ }^{2}$ Infrared Processing and Analysis Center, California Institute of Technology, Pasadena, CA 91125, USA \\ ${ }^{3}$ Department of Physics and Astronomy, UCLA, Los Angeles, CA 90095, USA \\ ${ }^{4}$ Department of Astronomy, University of Virginia, Charlottesville, VA 22904, USA \\ ${ }^{5}$ Department of Astronomy, Ohio State University, 140 West 18th Avenue, Columbus, OH 43210, USA \\ Received 2013 June 4; accepted 2014 February 4; published 2014 April 10
}

\begin{abstract}
As part of a larger search of Wide-field Infrared Survey Explorer (WISE) data for cool brown dwarfs with effective temperatures less than $1000 \mathrm{~K}$, we present the discovery of three new cool brown dwarfs with spectral types later than T7. Using low-resolution, near-infrared spectra obtained with the NASA Infrared Telescope Facility and the Hubble Space Telescope, we derive spectral types of T9.5 for WISE J094305.98+360723.5, T8 for WISE J200050.19+362950.1, and Y0: for WISE J220905.73+271143.9. The identification of WISE J220905.73+271143.9 as a Y dwarf brings the total number of spectroscopically confirmed Y dwarfs to 17. In addition, we present an improved spectrum (i.e., higher signal-to-noise ratio) of the Y0 dwarf WISE J041022.71+150248.4 that confirms the Cushing et al. classification of Y0. Spectrophotometric distance estimates place all three new brown dwarfs at distances less than 12 pc, with WISE J200050.19+362950.1 lying at a distance of only 3.9-8.0 pc. Finally, we note that brown dwarfs like WISE J200050.19+362950.1 that lie in or near the Galactic plane offer an exciting opportunity to directly measure the mass of a brown dwarf via astrometric microlensing.
\end{abstract}

Key words: brown dwarfs - infrared: stars - stars: individual (WISE J041022.71+150248.4, WISE J094305.98+360723.5, WISE J200050.19+362950.1, WISE J20905.73+271143.9) - stars: low-mass

Online-only material: color figures, supplemental data

\section{INTRODUCTION}

The launch of the Wide-field Infrared Survey Explorer (WISE; Wright et al. 2010) in late 2009 ushered in a new era in the study of brown dwarfs with spectral types later than T8 (effective temperatures $T_{\text {eff }}$ less than roughly $700 \mathrm{~K}$ ). Previous searches in the field with the Two Micron All Sky Survey (2MASS; Skrutskie et al. 2006; Burgasser et al. 2002, 2004), the UKIRT Infrared Deep Sky Survey (UKIDSS; Lawrence et al. 2007; Pinfield et al. 2008; Burningham et al. 2010), and the Canada France Brown Dwarf Survey (CFHBD; Delorme et al. 2008; Albert et al. 2011) uncovered 10 brown dwarfs with spectral types later than $\mathrm{T} 8$, but the survey sensitivity limits combined with their intrinsic faintness at red optical and near-infrared wavelengths $\left(M_{H} \gtrsim 19 \mathrm{mag}\right.$; Marsh et al. 2013) limited the ability of these surveys to identify large numbers of them.

In contrast, WISE surveyed the entire sky at four mid-infrared wavelengths centered at $3.4 \mu \mathrm{m}, 4.6 \mu \mathrm{m}, 12 \mu \mathrm{m}$, and $22 \mu \mathrm{m}$ (hereafter denoted $W 1, W 2, W 3$, and $W 4$, respectively) that are ideally suited to detect cool brown dwarfs because these bandpasses span the peak of their spectral energy distributions. In particular, the $W 1$ and $W 2$ bands are centered on a deep $\mathrm{CH}_{4}$ absorption band at $3.3 \mu \mathrm{m}$ and a region relatively free of opacity at $4.7 \mu \mathrm{m}$ where a large fraction of the total flux of cool brown dwarfs emerges (see Figure 2 of Mainzer et al. 2011). The resulting $W 1-W 2$ color is very red, which makes the identification of cool brown dwarf candidates relatively straightforward. To date, WISE data have been used to identify roughly 200 brown dwarfs with spectral types later than T6 (Mainzer et al. 2011; Burgasser et al. 2011; Scholz et al. 2011; Kirkpatrick et al. 2011, 2012; Cushing et al. 2011; Tinney et al. 2012; Luhman et al. 2012; Mace et al. 2013; Liu et al. 2011a; Luhman 2013; Thompson et al. 2013; Pinfield et al. 2014), including the 16 spectroscopically confirmed Y dwarfs previously published (Cushing et al. 2011; Kirkpatrick et al. 2012, 2013; Tinney et al. 2012; Liu et al. 2012).

The detailed study of these cool brown dwarfs promises to shed light on the physics of ultracool atmospheres and constrain brown dwarf formation theories. In particular, simulations by Burgasser (2004) suggest that the space density of cool brown dwarfs is very sensitive to the underlying mass function, a potential constraint on the yet unknown formation mechanism(s) of brown dwarfs (see Stamatellos 2013 for a review of the current theories). However, brown dwarfs obey a mass-luminosity-age relation, so measuring the brown dwarf present-day mass function is difficult given that the ages of the vast majority of brown dwarfs are unknown. The underlying mass function can be constrained, however, by first forward modeling the evolution of a population of brown dwarfs over time (e.g., Burgasser 2004; Allen et al. 2005; Deacon \& Hambly 2006) and then comparing the observed space densities of brown dwarfs to the predictions of the models. Given the relative ease with which effective temperatures can be estimated for cool brown dwarfs, the most common technique has been to compare the space densities of the brown dwarfs as a function of spectral type to model effective temperature distributions (e.g., Pinfield et al. 2008; Burningham et al. 2010, 2013; Kirkpatrick et al. 2012; Day-Jones et al. 2013). Results to date suggest that if the mass function is modeled as $d N / d M \propto M^{-\alpha}$, then $-1<\alpha<0$.

Implicit in this technique is the necessity of a complete, volume-limited sample of brown dwarfs. In this paper, we report three new discoveries from our ongoing program to construct 
Table 1

Photometry of New Brown Dwarfs

\begin{tabular}{|c|c|c|c|c|c|c|c|c|c|}
\hline \multirow[b]{2}{*}{ Object } & \multirow[b]{2}{*}{$\begin{array}{c}J \\
(\mathrm{mag})\end{array}$} & \multirow[b]{2}{*}{$\begin{array}{c}H \\
(\mathrm{mag})\end{array}$} & \multirow[b]{2}{*}{$\begin{array}{c}F 140 W^{\mathrm{b}} \\
(\mathrm{mag})\end{array}$} & \multicolumn{4}{|c|}{$W I S E^{\mathrm{a}}$} & \multicolumn{2}{|c|}{ Spitzer } \\
\hline & & & & $\begin{array}{c}\text { W1 } \\
\text { (mag) }\end{array}$ & $\begin{array}{c}\text { W2 } \\
\text { (mag) }\end{array}$ & $\begin{array}{c}\text { W3 } \\
\text { (mag) }\end{array}$ & $\begin{array}{c}\text { W4 } \\
\text { (mag) }\end{array}$ & $\begin{array}{l}{[3.6]} \\
\text { (mag) }\end{array}$ & $\begin{array}{l}{[4.5]} \\
(\mathrm{mag})\end{array}$ \\
\hline WISE J094305.98+360723.5 & $19.74 \pm 0.05$ & $20.37 \pm 0.20$ & $20.04 \pm 0.02$ & $>17.99$ & $14.37 \pm 0.06$ & $12.33 \pm 0.33$ & $>8.89$ & $16.75 \pm 0.04$ & $14.28 \pm 0.02$ \\
\hline WISE J200050.19+362950.1 & $15.44 \pm 0.01$ & $15.85 \pm 0.01$ & $\ldots$ & $15.56 \pm 0.09$ & $12.71 \pm 0.03$ & $11.26 \pm 0.11$ & $>9.55$ & $14.22 \pm 0.02$ & $12.68 \pm 0.02$ \\
\hline WISE J220905.73+271143.9 & $22.58 \pm 0.14$ & $22.98 \pm 0.31$ & $23.16 \pm 0.16$ & $>18.47$ & $14.79 \pm 0.07$ & $12.44 \pm 0.34$ & $>9.31$ & $17.82 \pm 0.09$ & $14.74 \pm 0.02$ \\
\hline
\end{tabular}

Notes. All $J$ - and $H$-band photometry is on the MKO-NIR system.

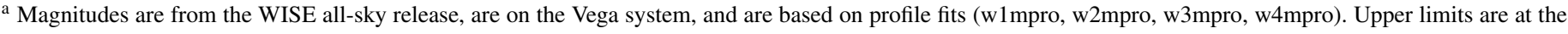
95\% confidence level (see http://wise2.ipac.caltech.edu/docs/release/allsky/expsup/sec4_4c.html\#ul2).

b WFC3/HST magnitudes are on the Vega system.

just such a volume-limited sample of cool brown dwarfs using data from WISE. In addition, we present an improved $J$ - and $H$-band spectrum of the Y0 dwarf WISE J041022.71+150248.4 (hereafter WISE J0410+1502) located at a distance of roughly 4 pc (Marsh et al. 2013).

\section{CANDIDATE SELECTION}

WISE J094305.98+360723.5, WISE J200050.19+362950.1, and WISE J220905.73+271143.9 (hereafter WISE J0943+3607, WISE J2000+3629, and WISE J2209+2711) were identified as candidate cold brown dwarfs as part of our larger search of the WISE All-Sky Catalog and All-Sky Reject Table. A description of the criteria used to select brown dwarf candidates can be found in Kirkpatrick et al. (2012). Briefly, the primary selection criterion is that the $W 1-W 2$ color from profile-fit photometry is greater than $2.0 \mathrm{mag}$, which corresponds roughly to a spectral type of T6. Table 1 lists the positions (as part of the WISE identification) and WISE photometry of the new brown dwarfs, and Figure 1 gives finder charts at both near- and mid-infrared wavelengths.

\section{OBSERVATIONS}

We have an extensive and ongoing observational follow-up campaign to confirm brown dwarf candidates identified using WISE data. In the following sections, we describe the nearand mid-infrared photometric and spectroscopic observations of WISE J0943+3607, WISE J0410+1502, WISE J2000+3629, and WISE J2209+2711.

\subsection{Near- and Mid-Infrared Imaging}

\subsubsection{UKIDSS}

WISE J2000+3629 was observed as part of the UKIDSS Galactic plane Survey (Lucas et al. 2008) in the Mauna Kea Observatories Near-Infrared (MKO-NIR; Simons \& Tokunaga 2002; Tokunaga et al. 2002; Tokunaga \& Vacca 2005) $J$ and $H$ bands on 2009 July 19 (UT). The $J$ - and $H$-band photometry of WISE J2000+3629 (sourceID 438792120568) was obtained from Data Release 7 using the WFCAM Science Archive and is given in Table 1.

\subsubsection{WIRC/Hale 200 inch}

WISE J0943+3607 was targeted with the Wide-field Infrared Camera (WIRC; Wilson et al. 2003) on the 200 inch Hale Telescope at Palomar Observatory. It was observed in the MKONIR $J$ and $H$ bands on the night of 2010 Dec 24 (UT). WIRC uses a $2048 \times 2048$ HAWAII-2 array with a plate scale of $0^{\prime \prime} .2487$ pixel $^{-1}$ resulting in a $8.7 \times 8.7$ field of view. We obtained fifteen $60 \mathrm{~s}$ exposures (900 s total) in each of the MKO-NIR $J$ and $H$ bands. A description of the data reduction can be found in Kirkpatrick et al. (2011). The $J$ - and $H$-band magnitudes of WISE J0943+3607 are given in Table 1.

\subsubsection{Keck II/NIRC2}

WISE J2209+2711 was observed with NIRC2 behind the Keck II LGS-AO system (Wizinowich et al. 2006; van Dam et al. 2006) on the night of 2011 July 20. We used the $R=$ 13.3 USNO-B star 1171-0655438 (Monet et al. 2003) located $23^{\prime \prime}$ from the target for the tip-tilt reference star. Images were obtained with the $J$ and $H$ MKO-NIR filters and the wide camera setting (nominal pixel scale $=0$ '.039686 pixel $^{-1}$ ), which provided a single-frame field of view of $40^{\prime \prime} \times 40^{\prime \prime}$. We stacked and median averaged $27 \mathrm{~J}$-band images (120 s exposure times) and $54 \mathrm{H}$-band images (60 s exposure times) to create $J$ and $H$ mosaics with total exposure times of 54 minutes. The mosaics were photometrically calibrated using two stars in common between the NIRC2 images and much wider field-of-view, though shallower $J$ and $H$ WIRC images.

\subsubsection{WFC3/HST}

Four images each of WISE J0943+3607 and WISE J2209+2711 through the $F 140 W$ filter $\left(\lambda_{p}=1392.3 \mathrm{~nm}\right.$, width $=384 \mathrm{~nm}$ ) were obtained with the Wide Field Camera 3 (WFC3; Kimble et al. 2008) on board the Hubble Space Telescope $(H S T)$ as a part of a Cycle 19 program to obtain grism spectroscopy of Y dwarf candidates (GO-12544, PI=Cushing). The WFC3 uses a $1024 \times 1024 \mathrm{HgCdTe}$ detector with a plate scale of 0 '. 13 pixel $^{-1}$, which results in a field of view of $123 \times$ 126 arcsecond. Each exposure was $78 \mathrm{~s}$ long for a total exposure time of $312 \mathrm{~s}$.

For each source, the four images were combined using MULTIDRIZZLE (Koekemoer et al. 2002). Photometry of each source was performed using a 0.'4 radius aperture. The noise in each pixel of a drizzled image is correlated so the rms noise in the local background (i.e., a sky annulus) cannot be used to estimate the noise in the background (e.g., Casertano et al. 2000). We therefore measured the background in an aperture of $00^{\prime \prime} 4$ at 1000 star-free positions on the image. The background level and uncertainty are given by the mean and standard deviation of the resulting distribution. The $F 140 \mathrm{~W}$ magnitudes of WISE J0943+3607 and WISE J2209+2711 on the Vega system are given in Table 1 and were computed using the F140W zero point of 25.1845 mag. ${ }^{6}$

\footnotetext{
6 http://www.stsci.edu/hst/wfc3/phot_zp_lbn
} 

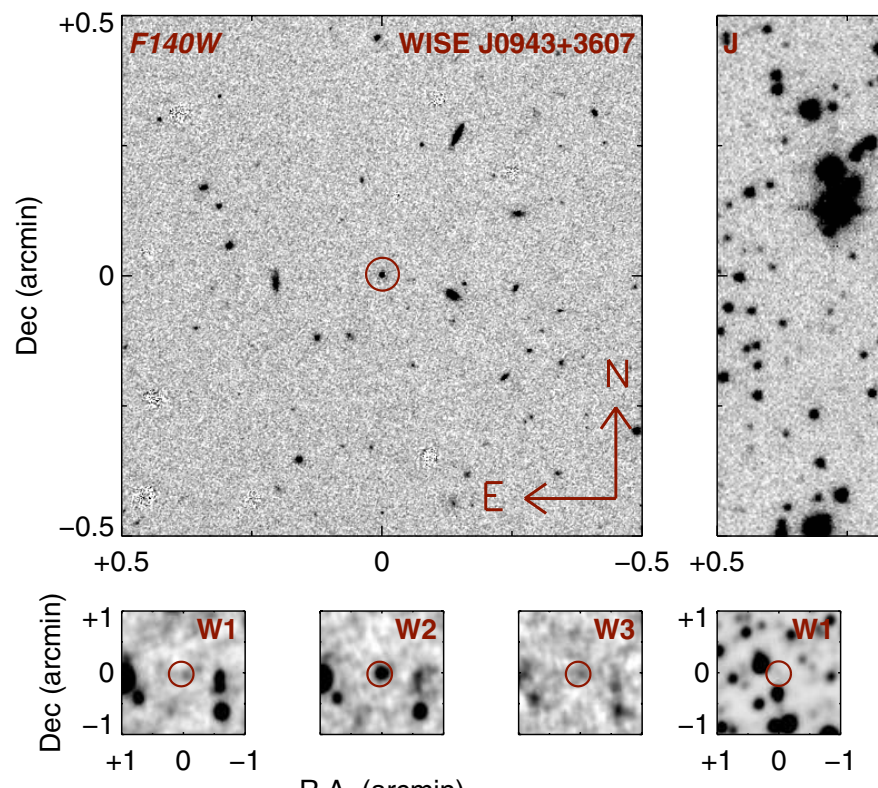

R.A. (arcmin)

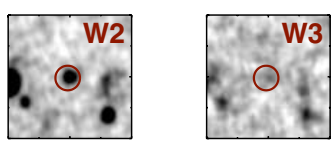

R.A. (arcmin)

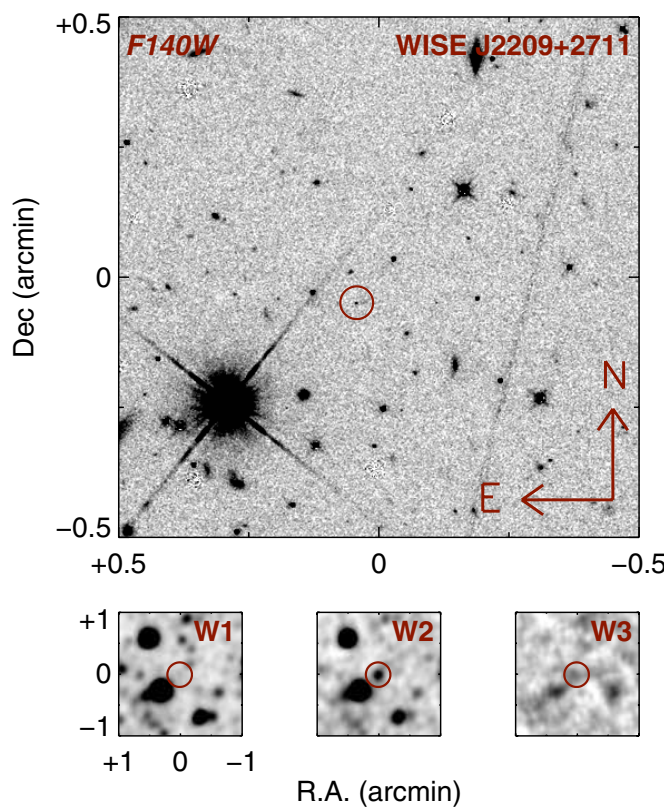

Figure 1. Finder charts for the new brown dwarf discoveries, WISE J0943+3607, WISE J2000+3629, and WISE J2209+2711. For each source, the lower three panels are $2 \times 2$ arcmin WISE $W 1, W 2$, and $W 3$ band images while the larger panel is a $1 \times 1$ arcmin near-infrared image. The near-infrared images for WISE J0943+3607 and WISE J2209+2711 were obtained through the $F 140 W$ filters of the WFC 3 on board $H S T$ while the image for WISE J2000+3629 is from UKIDSS and was obtained through the MKO-NIR $J$-band filter. All images are oriented such that north is up and east is to the left.

(A color version of this figure is available in the online journal.)

\subsubsection{IRAC/Spitzer}

WISE J0943+3607, WISE J2000+3629, and WISE J2209+2711 were observed with the Infrared Array Camera (IRAC; Fazio et al. 2004) on board the Spitzer Space Telescope (Werner et al. 2004) as part of Cycle 7 and Cycle 8 programs 70062 and 80109 (PI: Kirkpatrick). We used IRAC (Fazio et al. 2004) to image each dwarf in bandpasses centered at 3.6 and $4.5 \mu \mathrm{m}$ (hereafter denoted as [3.6] and [4.5]). These bandpasses are similar to the $W 1$ and $W 2$ bands (see Figure 2 of Mainzer et al. 2011) and therefore provide more sensitive measurements of the emergent flux at these wavelengths, particularly at $3.6 \mu \mathrm{m}$ where the WISE measurements are often upper limits. IRAC employs arrays with a pixel scale of 1 1".2 pixel $^{-1}$ that cover a field of view of $5.2 \times 5.2$. A description of the data acquisition and reduction for our Spitzer programs can be found in Kirkpatrick et al. (2011). The IRAC/Spitzer [3.6] and [4.5] mag of the three sources are given in Table 1. We note that Griffith et al. (2012) present photometry of WISE J0943+3607 and WISE J2209+2711, using the same data but a slightly different algorithm, and find similar magnitudes.

\subsection{Near-Infrared Spectroscopy

$$
\text { 3.2.1. SpeX/IRTF }
$$

A near-infrared spectrum of WISE J2000+3629 was obtained with SpeX (Rayner et al. 2003) on the $3.0 \mathrm{~m}$ NASA Infrared Telescope Facility (IRTF) atop Mauna Kea, H I on the night of 2012 May 28 (UT). The low-resolution prism mode was used in conjunction with a 0.5 wide slit, resulting in a spectrum that 
Table 2

Derived Properties of New Brown Dwarfs

\begin{tabular}{lccc}
\hline \hline Object & $\begin{array}{c}\text { Spectral } \\
\text { Type }\end{array}$ & $\begin{array}{c}M_{H} \text { Spectrophotometric } \\
\text { Distance }\end{array}$ & \multicolumn{2}{c}{ W2 Spectrophotometric } \\
& Distance & $6.6-12.1$ \\
WISE J0943+3607 & T9.5 & $7.9-34.3$ & $6.4-8.0$ \\
WISE J2000+3629 & T8 & $3.9-7.3$ & $5.2-11.7$ \\
WISE J2209+2711 & Y0: & $\cdots$ & \\
\hline
\end{tabular}

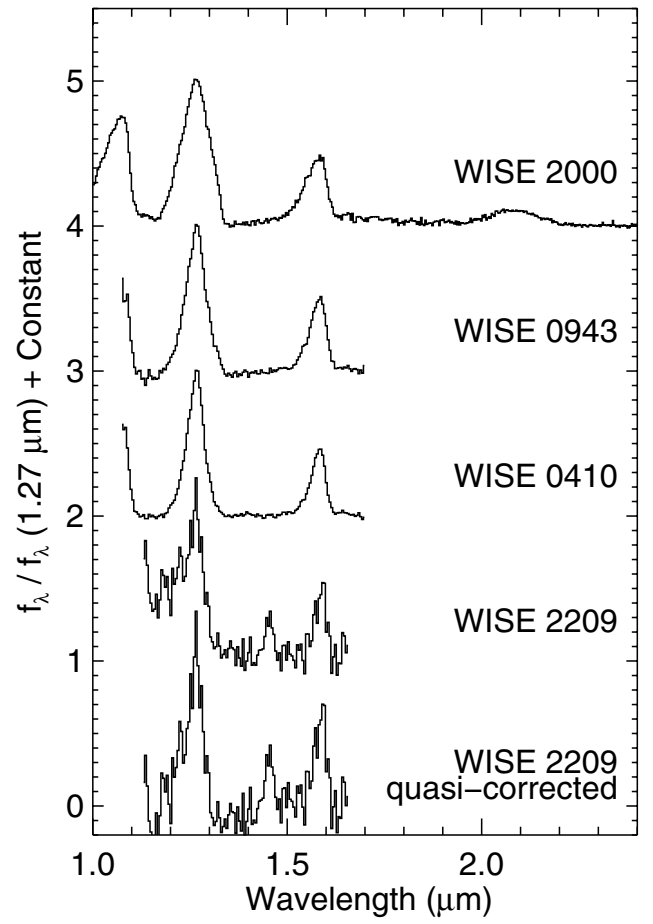

Figure 2. Near-infrared spectra of WISE J2000+3629, WISE J0943+3607, WISE J0410+1502, and WISE J2209+2711. The spectrum of WISE J2209+2711 was contaminated, so we subtracted a low-order polynomial as described in the text to produce a quasi-corrected spectrum (bottom spectrum).

(Supplemental data of this figure are available in the online journal.)

covered the wavelength range $0.8-2.5 \mu \mathrm{m}$ at a resolving power of $\sim 150$. A series of ten $120 \mathrm{~s}$ exposures were obtained at two different positions along the $15^{\prime \prime}$ long slit for a total exposure time of $1200 \mathrm{~s}$. The A0 V star HD 192538 was observed for telluric correction and flux calibration and argon arc and quartztungsten exposures for wavelength calibration and flat-fielding purposes.

The data were reduced using Spextool, the IDL-based data reduction package for SpeX (Cushing et al. 2004). Pairs of exposures taken at the different slit positions were corrected for nonlinearity, pair-subtracted, and then flat-fielded. The raw spectra were then optimally extracted after subtracting any residual background and wavelength calibrated using the argon arc spectrum. The 10 spectra were then combined using a weighted average. Telluric correction and flux calibration were accomplished using the spectrum of HD 192538 and the technique described by Vacca et al. (2003). The final spectrum is shown in Figure 2 and has signal-to-noise ratios of 75, 110, 40 , and 20 at the peaks of the $Y, J, H$, and $K$ bands, respectively.

\subsection{2. $W F C 3 / H S T$}

WISE J0410+1502, WISE J0943+3607, and WISE J2209+2711 were observed with the infrared channel of the
WFC3 (Kimble et al. 2008) on board the HST as part of a Cycle 19 program (GO-12544, PI = Cushing). The G141 grism was used to perform slitless spectroscopy of each brown dwarf covering the $1.07-1.70 \mu \mathrm{m}$ wavelength range at a resolving power of $R \approx 130$. A description of the data acquisition and reduction can be found in Cushing et al. (2011). The final signalto-noise ratio of the WISE J2209+2711 spectrum is low, with a peak of $\sim 6$ in the $J$ band and $\sim 5$ in the $H$ band, while that of WISE J0943+3607 and WISE J0410+1502 is $\gtrsim 30$ at the peaks of the $J$ and $H$ bands.

Spectroscopy with WFC3 is achieved via a slitless grism, so some of the resulting spectra could be contaminated by light from other stars in the field. Such is the case for WISE J2209+2711. The contaminated spectrum of WISE J2209+2711 is shown in Figure 2 and exhibits a smooth rise in flux toward shorter wavelengths due to contaminating light of another star in the field. We therefore subtracted a second-order polynomial fit to the flux densities in the $1.1-1.2 \mu \mathrm{m}, 1.3-1.5 \mu \mathrm{m}$, and $1.62-1.68 \mu \mathrm{m}$ wavelength intervals in order to produce a quasi-corrected spectrum, which is also shown in Figure 2.

\section{ANALYSIS}

The spectral types of WISE J0410+1502, WISE J0943+3607, WISE J2000+3629, and WISE J2209+2711 were determined by direct comparison to the $\mathrm{T}$ and $\mathrm{Y}$ dwarf spectral standards of Burgasser et al. (2006), Cushing et al. (2011), and Kirkpatrick et al. (2012). Figures 3 and 4 show the near-infrared spectra of each brown dwarf plotted over the late-type $\mathrm{T}$ and $\mathrm{Y}$ dwarf spectral standards. The final spectral types are given in Table 2 and a brief discussion of some salient details regarding the classification of these brown dwarfs is given below.

WISE J0410+1502 is one of the six original Y dwarfs presented by Cushing et al. (2011). The Cushing et al. spectrum obtained with the Folded-port InfraRed Echellette (FIRE; Simcoe et al. 2010) spectrograph at the $6.5 \mathrm{~m}$ Magellan Baade Telescope covers the $1.0-2.4 \mu \mathrm{m}$ wavelength range at $R=$ 250-350, but the signal-to-noise ratio was very low, reaching a peak value of only $\sim 6$ in the $J$ band. WISE J0410+1502 was classified as Y0 based primarily on the width of its $J$-band peak. The higher signal-to-noise ratio HST/WFC3 spectrum presented herein covers only the $1.10-1.65 \mu \mathrm{m}$ wavelength range but confirms a spectral type of $\mathrm{Y} 0$ as it is a near perfect match to the Y0 spectral standard WISE J173835.53+273259.0 (left panel of Figure 3).

The quasi-corrected spectrum of WISE J2209+2711 is shown in the right panel of Figure 4. Based on the width of its $J$-band peak, WISE J2209+2711 is clearly later than T9. The Y0 spectral standard is a reasonable match but the low signal-to-noise ratio of the spectrum makes it difficult to determine whether it could be as late as Y1, so we classify WISE J2209+2711 as Y0:. An uncontaminated and higher signal-to-noise ratio spectrum will be required for a more precise spectral type. 

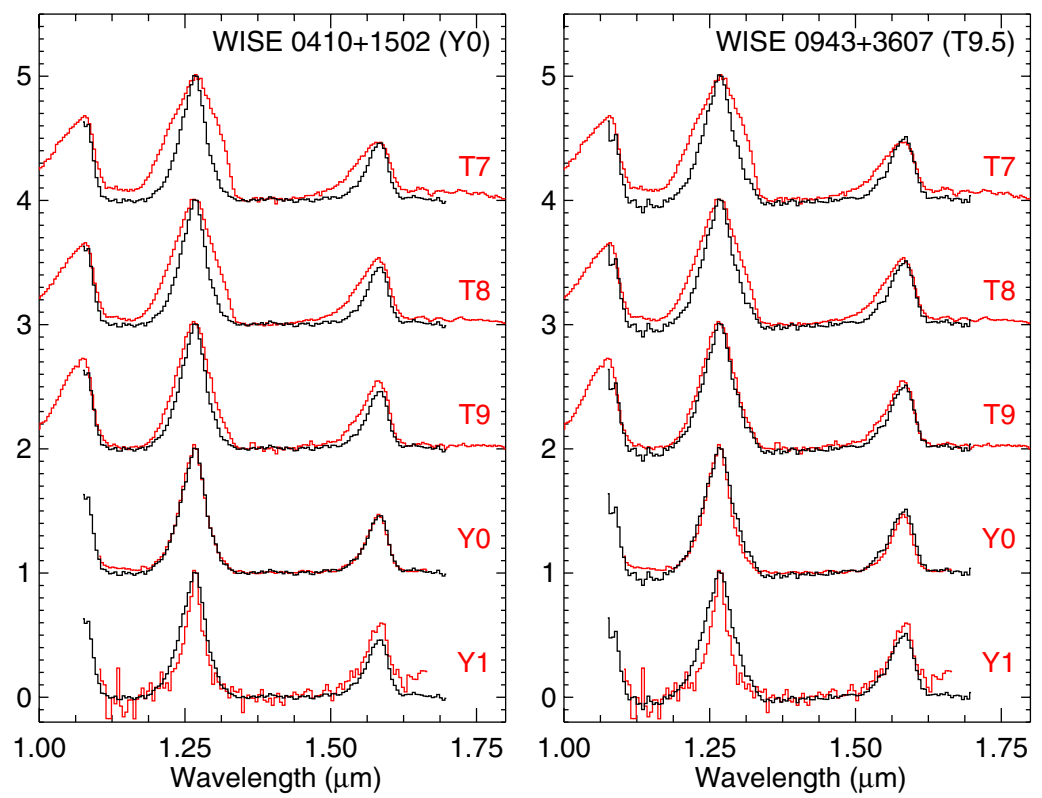

Figure 3. Spectral classification of WISE J0410+1502 and WISE J0943+3607 by direct spectral comparison. The late-type T dwarf and tentative Y dwarf spectral standards from Burgasser et al. (2006), Cushing et al. (2011), and Kirkpatrick et al. (2012) are shown in red, while the spectra of the unclassified brown dwarfs are shown in black.

(A color version of this figure is available in the online journal.)
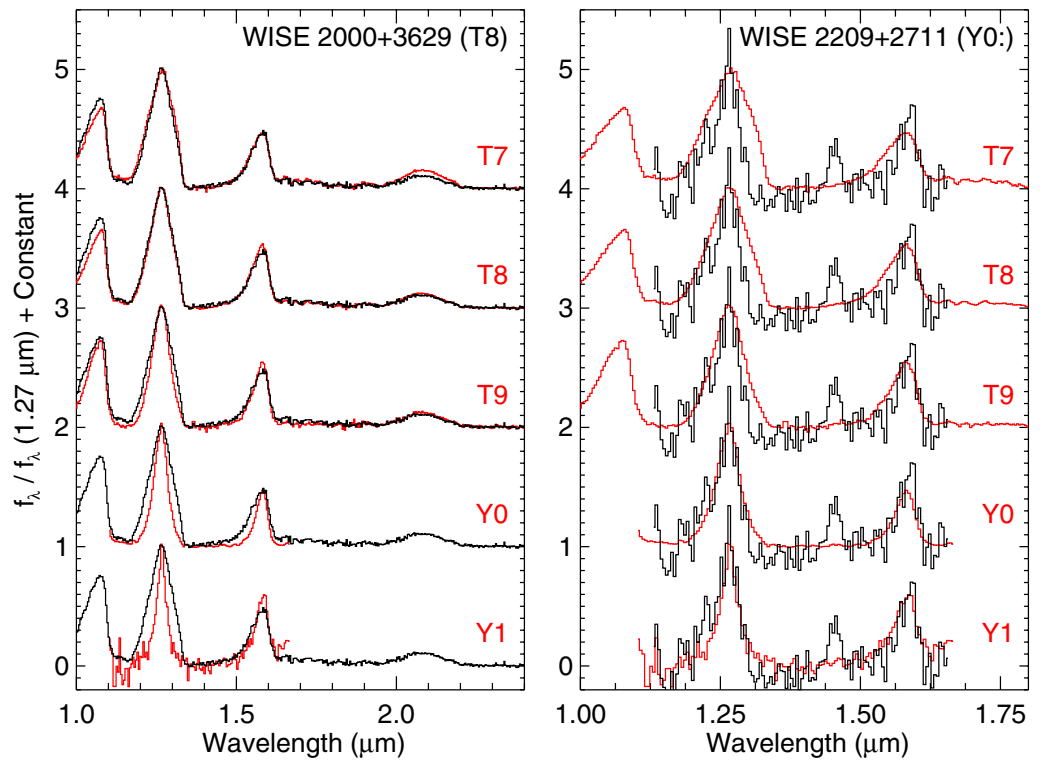

Figure 4. Spectral classification of WISE J2000+3629 and WISE J2209+2711 by direct spectral comparison. The late-type T dwarf and tentative Y dwarf spectral standards from Burgasser et al. (2006), Cushing et al. (2011), and Kirkpatrick et al. (2012) are shown in red, while the spectra of the unclassified brown dwarfs are shown in black.

(A color version of this figure is available in the online journal.)

Central to the construction of a volume-limited sample is accurate knowledge of the distances to the nearby stars and brown dwarfs. The new brown dwarfs presented herein are currently being observed as part of our program to measure parallaxes to the sample of cold brown dwarfs in the solar neighborhood. We can nevertheless derive spectrophotometric distance estimates to them using the $H$ and $W 2$ absolute magnitude spectral type relations of Kirkpatrick et al. (2012). ${ }^{7}$ The range of possible distances for each bandpass is given in Table 2 and includes the

7 Marsh et al. (2013) rederived both the $M_{H}$ and $W 2$ versus spectral type relations with updated distances but found similar results to Kirkpatrick et al. (2012). half subclass uncertainty in the spectral types and the photometric uncertainties. The $W 2$ spectrophotometric distance estimates place the three new brown dwarfs at distances between 6 and $12 \mathrm{pc}$. WISE J2000+3629 lies at a distances of 3.9-7.3 pc based on its $H$-band magnitude and a distance of $6.4-8.0 \mathrm{pc}$ based on its $W 2$ magnitude. We therefore estimate its distance to be $3.9-8.0 \mathrm{pc}$, which places it within the 8 pc sample.

\section{DISCUSSION}

The classification of WISE J2209+2711 as a Y dwarf brings the total number of spectroscopically confirmed Y dwarfs 


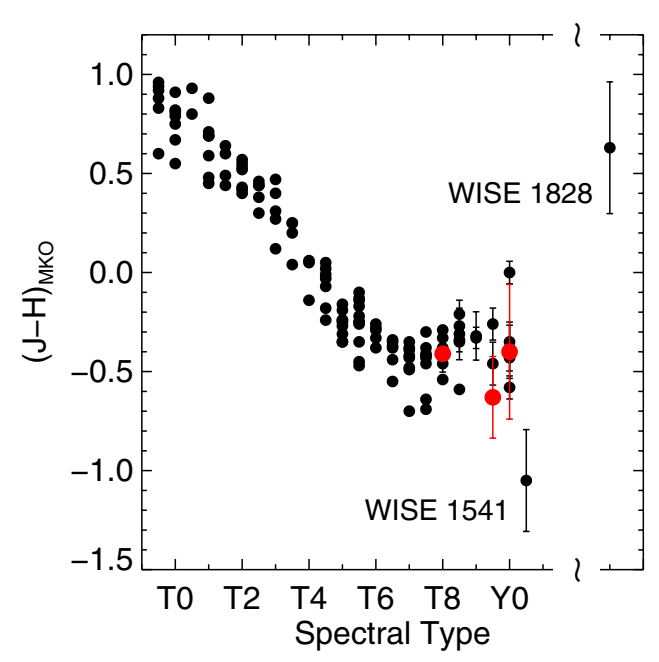

Figure 5. MKO-NIR $J-H$ colors of $\mathrm{T}$ and $\mathrm{Y}$ dwarfs as a function of spectral type. The filled black circles are from Leggett et al. (2010, 2013), while the red filled circles are from this work. WISE J182831.08+265037.7 is plotted separately because its spectral type is currently $\geqslant Y 2$. The three new brown dwarfs fall in line with the sequence, suggesting they are not peculiar.

(A color version of this figure is available in the online journal.)

to $17 .{ }^{8}$ The colors of the late-type $\mathrm{T}$ dwarfs and $\mathrm{Y}$ dwarfs exhibit distinct changes at the $\mathrm{T} / \mathrm{Y}$ boundary with the $J-H$ colors plateauing (albeit with a large scatter; Cushing et al. 2011; Kirkpatrick et al. 2012) and the $z^{\prime}-J, z^{\prime}-H$, and $Y-J$ colors rapidly becoming bluer (Liu et al. 2012; Lodieu et al. 2013). Figure 5 shows the MKO $J-H$ colors of a sample of $\mathrm{T}$ and Y dwarfs (Leggett et al. 2010, 2013) as a function of spectral type. The $J-H$ colors plateau in the late-T spectral types and turn redward at later types (with the exception of the Y0.5 dwarf WISE J154151.65-225024.9). The three new brown dwarfs are shown as filled red circles and fall in line with the known sequence, suggesting that they are not peculiar.

The identification of three new late-type brown dwarfs that lie within roughly $10 \mathrm{pc}$ of the Sun is an important addition to the sample of nearby cold brown dwarfs. We do, however, defer updating the space density estimates of the late-type $\mathrm{T}$ dwarfs and $\mathrm{Y}$ dwarfs presented until the parallaxes of these brown dwarfs have been measured and more nearby cool brown dwarfs are identified.

One of the three new brown dwarfs, WISE J2000+3629, is located in a region $(l=72.7, b=+3.32)$ covered by the UKIDSS Galactic Plane Survey (Lucas et al. 2008). Lucas et al. (2010) searched the Sixth Data Release of the Galactic Plane Survey to search for late-type $\mathrm{T}$ or $\mathrm{Y}$ dwarf candidates, but identified only a single candidate-the wellknown T9 dwarf UGPS J072227.51-054031.2. The position of WISE J2000+3629 was not covered by the Sixth Data Release but even if it had been, WISE J2000+3629 would have been missed by Lucas et al. (2010) because its values of $k_{-} 1 \mathrm{Ell}$ and jppErrbits, two flags in the UKIDSS catalog that describe the source ellipticity and photometric quality, fall outside of the Lucas et al. search criteria. This suggests that perhaps more brown dwarfs in the Galactic plane await discovery.

Indeed, WISE J2000+3629 joins a growing list of brown dwarfs identified in or near the Galactic plane, includ-

\footnotetext{
8 WD 0806-661 (Luhman et al. 2011) and CFBDSIR J1458+1013B (Liu et al. 2011b) have estimated effective temperatures similar to those of the known population of $\mathrm{Y}$ dwarfs but spectra of these cold brown dwarfs have yet
} to be obtained due to their faintness. ing UGPS J072227.51-054031.2 and Luhman's binary system WISE J104915.57-531906.1 (Luhman 2013). Previous searches for brown dwarfs including our own ignore either part of, or the entire, Galactic plane in order to minimize the impact of stellar contamination (e.g., Burgasser et al. 2002; Cruz et al. 2007, but see Folkes et al. 2007, 2012). However, once a brown dwarf has been discovered in the plane, the reasonably high surface density of background stars offers us an opportunity to measure the mass of the brown dwarf via astrometric microlensing (Refsdal 1964; Paczyński 1995; Miralda-Escude 1996).

During a microlensing event, the lens (i.e., the brown dwarf) both magnifies the source (i.e., the background star) and shifts its centroid. If the angular separation between the lens and the source is much larger than the Einstein radius, then the magnification is negligible but the centroid shift remains (e.g., Miralda-Escude 1996; Gould 2001). If the lens is also dark (i.e., is much fainter than the source), then a measurement of the centroid shift of the source is relatively straightforward and yields the mass of the lens. Cool $\mathrm{T}$ and $\mathrm{Y}$ dwarfs are ideal targets for astrometric microlensing measurements at optical wavelengths because they are effectively dark at these wavelengths $\left(M_{V} \gtrsim 26 \mathrm{mag}\right.$; King et al. 2010; Boffin et al. 2013). The expected number of lensing events per year, which is derived in the Appendix, is given by

$$
N=2 \kappa \pi_{L} \mu_{L} \sigma_{\mathrm{bg}}\left(\frac{\delta M_{L}}{\delta \Delta \theta}\right),
$$

where $\kappa \equiv\left(4 G / c^{2} \mathrm{AU}\right) \approx 8.144$ mas $/ M_{\odot}, \pi_{L}$ is the parallax of the lens in units of arcseconds, $\mu_{L}$ is the proper motion of the lens in units of arcseconds per year, $\sigma_{\mathrm{bg}}$ is the surface density of stars in units of stars per square arcseconds, $\delta M_{L}$ is the required precision of the mass determination in solar masses, and $\delta \Delta \theta$ is the precision of the astrometric measurement in milliarcseconds.

Although we cannot compute a rate for WISE J2000+3629 because its parallax and proper motion are unknown, we can get a sense of the expected rate of brown dwarf lensing events near the Galactic plane by considering a hypothetical brown dwarf located at $l=45^{\circ}, b=0^{\circ}$, at a distance of $10 \mathrm{pc}$, and with a proper motion of $0.5 \mathrm{yr}^{-1}$. We used the Besançon model of the Galaxy (Robin et al. 2003) to generate a simulated population of stars in a $0.1 \times 0.1$ field at this position, which results in a surface density of stars with $V<24$ of 0.12 stars per square arcsecond. If we require a mass precision of $\delta M_{L}=0.10 M_{\odot}$ and assume we can achieve an astrometric precision of $\delta \Delta \theta=$ 0.2 mas (e.g., with the Hubble Space Telescope), then we find that $N \approx 0.05$, or roughly one event every two decades. The potential to measure the masses of even a handful of cool field brown dwarfs on a decades timescale should therefore stoke interest in searches for brown dwarfs in the zone of avoidance.

Computing values for UGPS J072227.51-054031.2 and Luhman's binary in a similar fashion, we find 0.02 and 0.3 , respectively. The value for Luhman's binary is remarkably high, one event every few years, because of its close distance $(\sim 2 \mathrm{pc})$ and high proper motion $\left(2\right.$ ". $\left.7 \mathrm{yr}^{-1}\right)$. Follow-up observations to determine whether it will actually lens a nearby star should therefore be a high priority since a mass measurement of such a nearby (binary) brown dwarf would be invaluable.

M.C.C. acknowledges fruitful discussions with Adam Schneider. We thank P. Eisenhardt for obtaining the NIRC2 data for WISE J0943+3607. Data presented herein were obtained at the 
W. M. Keck Observatory from telescope time allocated to the National Aeronautics and Space Administration through the agency's scientific partnership with the California Institute of Technology and the University of California. The Observatory was made possible by the generous financial support of the W. M. Keck Foundation. This publication makes use of data products from the Wide-field Infrared Survey Explorer, which is a joint project of the University of California, Los Angeles, and the Jet Propulsion Laboratory/California Institute of Technology, funded by the National Aeronautics and Space Administrations, the Two Micron All Sky Survey, which is a joint project of the University of Massachusetts and the Infrared Processing and Analysis Center/California Institute of Technology, funded by the National Aeronautics and Space Administration and the National Science Foundation, and is based [in part] on observations made with the Spitzer Space Telescope, which is operated by the Jet Propulsion Laboratory, California Institute of Technology, under a contract with NASA. Support for this work was provided by NASA through an award issued by JPL/Caltech and the NASA/ESA Hubble Space Telescope, obtained at the Space Telescope Science Institute, which is operated by the Association of Universities for Research in Astronomy, Inc., under NASA contract NAS 5-26555. The HST observations are associated with program \#12544, support for which was provided by NASA through a grant from the Space Telescope Science Institute, which is operated by the Association of Universities for Research in Astronomy, Inc., under NASA contract NAS 526555. This research has made use of the NASA/IPAC Infrared Science Archive, which is operated by the Jet Propulsion Laboratory, California Institute of Technology, under contract with the National Aeronautics and Space Administration. M.C.C. was a visiting Astronomer at the Infrared Telescope Facility, which is operated by the University of Hawaii under Cooperative Agreement No. NNX-08AE38A with the National Aeronautics and Space Administration, Science Mission Directorate, Planetary Astronomy Program. This research made use of the OSX Version of SCISOFT assembled by Dr. Nor Pirzkal and F. Pierfederici. A.G. was supported in part by NSF AST-1103471.

\section{APPENDIX}

The rate at which we can expect a nearby brown dwarf to astrometrically lens a background star can be calculated as follows. Let $\theta_{\max }$ be the maximum projected angle separation between the lens (i.e., the brown dwarf) and the source (i.e., a background star) for which we can measure the mass of the lens to a fixed precision via astrometric microlensing. The number of such events per year is then given by

$$
N=2 \theta_{\max } \mu_{\mathrm{L}} \sigma_{\mathrm{bg}}
$$

where $\mu_{L}$ is the proper motion of the lens in units of arcseconds per year (and thus $2 \theta_{\max } \mu_{L}$ is the total area swept out by the lens in a year) and $\sigma_{\mathrm{bg}}$ is the surface density of background stars in units of stars per square arcsecond. An expression for $\theta_{\max }$ can be derived thusly. Let $\theta$ be the projected angular separation between the lens and the source and $\theta_{E}$ be the angular Einstein radius given by

$$
\theta_{\mathrm{E}}=\sqrt{\frac{4 G M_{L}}{c^{2}} \frac{D_{S}-D_{L}}{D_{S} D_{L}}},
$$

where $M_{L}$ is the mass the lens, $D_{S}$ is the distance to the source, $D_{L}$ is the distance to the lens, and the other constants have their normal meaning. In the limit that $\theta \gg \theta_{\mathrm{E}}$, the angular shift in the centroid of the source for a dark lens (i.e., assuming the brown dwarf is much fainter than the source) is given by (Dominik \& Sahu 2000)

$$
\Delta \theta=\frac{\theta_{\mathrm{E}}^{2}}{\theta} .
$$

In the limit where $D_{S} \gg D_{L}$, the Einstein radius can be written as

$$
\theta_{\mathrm{E}}=\sqrt{\frac{4 G M_{L}}{c^{2} D_{L}}},
$$

and Equation (A3) becomes

$$
\Delta \theta=\frac{4 G M_{L}}{c^{2} \theta D_{L}}
$$

Taking the differential of this equation, holding $\theta$ and $D_{L}$ constant, we have

$$
\delta \Delta \theta=\frac{4 G \delta M_{L}}{c^{2} \theta D_{L}} .
$$

If we define $\theta_{\max }$ as the maximum projected angular separation between the lens and source, given $\delta M_{L}$, the required precision of the mass determination, and $\delta \Delta \theta$, the precision of the astrometric measurement, we have

$$
\theta_{\max }=\frac{4 G \delta M_{L}}{c^{2} D_{L} \delta \Delta \theta} .
$$

Define $\kappa$, a constant commonly used in the microlensing literature (e.g., Gould 2000), as

$$
\kappa \equiv \frac{4 G}{c^{2} \mathrm{AU}} \approx 8.144 \frac{\mathrm{mas}}{M_{\odot}},
$$

and we can rewrite Equation (A7) as

$$
\theta_{\max }=\frac{\kappa \delta M_{L} \pi_{L}}{\delta \Delta \theta},
$$

where $\pi_{L}$ is the parallax of the lens measured in arcseconds, and $\delta M_{L}$ and $\delta \Delta \theta$ are now measured in solar masses and milliarcseconds, respectively. Substituting into Equation (A1) we have for the number of astrometric microlensing event per year:

$$
N=2 \kappa \pi_{L} \mu_{L} \sigma_{\mathrm{bg}}\left(\frac{\delta M_{L}}{\delta \Delta \theta}\right) .
$$

\section{REFERENCES}

Albert, L., Artigau, É., Delorme, P., et al. 2011, AJ, 141, 203

Allen, P. R., Koerner, D. W., Reid, I. N., \& Trilling, D. E. 2005, ApJ, 625, 385 Boffin, H. M. J., Pourbaix, D., Mužić, K., et al. 2014, A\&A, 561, L4 Burgasser, A. J. 2004, ApJS, 155, 191

Burgasser, A. J., Cushing, M. C., Kirkpatrick, J. D., et al. 2011, ApJ, 735, 116 Burgasser, A. J., Geballe, T. R., Leggett, S. K., Kirkpatrick, J. D., \& Golimowski, D. A. 2006, ApJ, 637, 1067

Burgasser, A. J., Kirkpatrick, J. D., Brown, M. E., et al. 2002, ApJ, 564, 421 Burgasser, A. J., McElwain, M. W., Kirkpatrick, J. D., et al. 2004, AJ, 127, 2856 Burningham, B., Cardoso, C. V., Smith, L., et al. 2013, MNRAS, 433, 457 Burningham, B., Pinfield, D. J., Lucas, P. W., et al. 2010, MNRAS, 406, 1885 Casertano, S., de Mello, D., Dickinson, M., et al. 2000, AJ, 120, 2747 Cruz, K. L., Reid, I. N., Kirkpatrick, J. D., et al. 2007, AJ, 133, 439 Cushing, M. C., Kirkpatrick, J. D., Gelino, C. R., et al. 2011, ApJ, 743, 50 Cushing, M. C., Vacca, W. D., \& Rayner, J. T. 2004, PASP, 116, 362 Day-Jones, A. C., Marocco, F., Pinfield, D. J., et al. 2013, MNRAS, 430, 1171 Deacon, N. R., \& Hambly, N. C. 2006, MNRAS, 371, 1722 Delorme, P., Willott, C. J., Forveille, T., et al. 2008, A\&A, 484, 469 
Dominik, M., \& Sahu, K. C. 2000, ApJ, 534, 213

Fazio, G. G., Hora, J. L., Allen, L. E., et al. 2004, ApJS, 154, 10

Folkes, S. L., Pinfield, D. J., Jones, H. R. A., et al. 2012, MNRAS, 427, 3280

Folkes, S. L., Pinfield, D. J., Kendall, T. R., \& Jones, H. R. A. 2007, MNRAS, 378,901

Gould, A. 2000, ApJ, 542, 785

Gould, A. 2001, PASP, 113, 903

Griffith, R. L., Kirkpatrick, J. D., Eisenhardt, P. R. M., et al. 2012, AJ, 144, 148

Kimble, R. A., MacKenty, J. W., O'Connell, R. W., \& Townsend, J. A. 2008, Proc. SPIE, 7010, 70101

King, R. R., McCaughrean, M. J., Homeier, D., et al. 2010, A\&A, 510, A99

Kirkpatrick, J. D., Cushing, M. C., Gelino, C. R., et al. 2011, ApJS, 197, 19

Kirkpatrick, J. D., Cushing, M. C., Gelino, C. R., et al. 2013, ApJ, 776, 128

Kirkpatrick, J. D., Gelino, C. R., Cushing, M. C., et al. 2012, ApJ, 753, 156

Koekemoer, A. M., Fruchter, A. S., Hook, R. N., \& Hack, W. 2002, in The 2002 HST Calibration Workshop: Hubble after the Installation of the ACS and the NICMOS Cooling System, ed. S. Arribas, A. Koekemoer, \& B. Whitmore (Baltimore, MD: STScI), 337

Lawrence, A., Warren, S. J., Almaini, O., et al. 2007, MNRAS, 379, 1599

Leggett, S. K., Burningham, B., Saumon, D., et al. 2010, ApJ, 710, 1627

Leggett, S. K., Morley, C. V., Marley, M. S., et al. 2013, ApJ, 763, 130

Liu, M. C., Deacon, N. R., Magnier, E. A., et al. 2011a, ApJL, 740, L32

Liu, M. C., Delorme, P., Dupuy, T. J., et al. 2011b, ApJ, 740, 108

Liu, M. C., Dupuy, T. J., Bowler, B. P., Leggett, S. K., \& Best, W. M. J. 2012, ApJ, 758,57

Lodieu, N., Béjar, V. J. S., \& Rebolo, R. 2013, A\&A, 550, L2

Lucas, P. W., Hoare, M. G., Longmore, A., et al. 2008, MNRAS, 391, 136

Lucas, P. W., Tinney, C. G., Burningham, B., et al. 2010, MNRAS, 408, L56

Luhman, K. L. 2013, ApJL, 767, L1

Luhman, K. L., Burgasser, A. J., \& Bochanski, J. J. 2011, ApJL, 730, L9

Luhman, K. L., Loutrel, N. P., McCurdy, N. S., et al. 2012, ApJ, 760, 152
Mace, G. N., Kirkpatrick, J. D., Cushing, M. C., et al. 2013, ApJS, 205, 6 Mainzer, A., Michael, C., Skrutskie, M., et al. 2011, ApJ, 726, 30

Marsh, K. A., Wright, E. L., Kirkpatrick, J. D., et al. 2013, ApJ, 762, 119

Miralda-Escude, J. 1996, ApJL, 470, L113

Monet, D. G., Levine, S. E., Canzian, B., et al. 2003, AJ, 125, 984

Paczyński, B. 1995, AcA, 45, 345

Pinfield, D. J., Burningham, B., Tamura, M., et al. 2008, MNRAS, 390, 304

Pinfield, D. J., Gomes, J., Day-Jones, A. C., et al. 2014, MNRAS, 437, 1009

Rayner, J. T., Toomey, D. W., Onaka, P. M., et al. 2003, PASP, 115, 362

Refsdal, S. 1964, MNRAS, 128, 295

Robin, A. C., Reylé, C., Derrière, S., \& Picaud, S. 2003, A\&A, 409, 523

Scholz, R.-D., Bihain, G., Schnurr, O., \& Storm, J. 2011, A\&A, 532, L5

Simcoe, R. A., Burgasser, A. J., Bochanski, J. J., et al. 2010, Proc. SPIE, 7735 773514

Simons, D. A., \& Tokunaga, A. 2002, PASP, 114, 169

Skrutskie, M. F., Cutri, R. M., Stiening, R., et al. 2006, AJ, 131, 1163

Stamatellos, D. 2014, in The Labyrinth of Star Formation, ed. D. Stamatellos, S. Goodwin, \& D. Ward-Thompson (New York: Springer), in press (arXiv:1302.3954)

Thompson, M. A., Kirkpatrick, J. D., Mace, Gregory N., et al. 2013, PASP, 125,809

Tinney, C. G., Faherty, J. K., Kirkpatrick, J. D., et al. 2012, ApJ, 759, 60

Tokunaga, A. T., Simons, D. A., \& Vacca, W. D. 2002, PASP, 114, 180

Tokunaga, A. T., \& Vacca, W. D. 2005, PASP, 117, 421

Vacca, W. D., Cushing, M. C., \& Rayner, J. T. 2003, PASP, 115, 389

van Dam, M. A., Bouchez, A. H., Le Mignant, D., et al. 2006, PASP, 118, 310

Werner, M. W., Roellig, T. L., Low, F. J., et al. 2004, ApJS, 154, 1

Wilson, J. C., Eikenberry, S. S., Henderson, C. P., et al. 2003, Proc. SPIE, 4841, 451

Wizinowich, P. L., Le Mignant, D., Bouchez, A. H., et al. 2006, PASP, 118, 297

Wright, E. L., Eisenhardt, P. R. M., Mainzer, A. K., et al. 2010, AJ, 140, 1868 